\title{
Gestión de la calidad en una unidad de cuidados intensivos: implementación de la norma ISO 9001:2008
}

\author{
R. Lorenzo Torrent ${ }^{a}$, M. Sánchez Palacios ${ }^{a}$, L. Santana Cabrera ${ }^{a, *}$, J.L. Cobian Martinez ${ }^{b}$ y \\ C. García del Rosario ${ }^{\mathrm{b}}$
}

aServicio de Medicina Intensiva, Hospital Universitario Insular de Gran Canaria, Gran Canaria, España
'Unidad de Calidad del Complejo Hospitalario Universitario Insular de Gran Canaria, Gran Canaria, España

Recibido el 5 de junio de 2009; aceptado el 18 de junio de 2009

Disponible en Internet el 25 de septiembre de 2009

\section{PALABRAS CLAVE \\ Control de calidad; Calidad de la atención sanitaria; \\ Cuidados intensivos}

\section{KEYWORDS}

Quality control;

Quality of health

care;

Intensive care

\begin{abstract}
Resumen
Los sistemas de gestión de la calidad permiten priorizar acciones para que se pueda mantener la seguridad y la eficacia de las tecnologías sanitarias. En la Unidad de Cuidados Intensivos ( $\mathrm{UCl}$ ) de nuestro hospital se ha implementado un plan de gestión de la calidad, que ha obtenido el reconocimiento como "Servicio Certificado que gestiona sus actividades según la norma UNE-EN ISO 9001:2008”. Con la aplicación del sistema de gestión de la calidad se logra detectar las necesidades que puede cubrir el servicio, esto con el fin de lograr la satisfacción del paciente, familiar o personal sanitario del resto de los servicios del hospital, mejorar las comunicaciones dentro y fuera del servicio, conseguir una mayor comprensión de los procesos de la organización y control de riesgo, delimitar responsabilidades de forma clara a todo el personal, llevar a cabo una mejor utilización del tiempo y los recursos y, por último, mejorar, si cabe, la motivación del personal. (c) 2009 Elsevier España, S.L. y SEMICYUC. Todos los derechos reservados.
\end{abstract}

Management of Quality in an Intensive Care Unit: Implementation of ISO 9001:2008 international standard

\begin{abstract}
The Quality Management Systems make it possible to prioritize actions to maintain the safety and efficacy of health technologies. The Intensive Care Unit of our hospital has implemented a quality management plan, which has obtained accreditation as "Service Certificate that manages its activities according to UNE-EN ISO 9001:2008" standard. With the application of quality management system, it has been possible to detect the needs that the Service can cover in order to obtain the satisfaction of the patient, relative or health personnel of the other services of the hospital, to improve communications inside
\end{abstract}

\footnotetext{
*Autor para correspondencia.

Correo electrónico: Isancabx@gobiernodecanarias.org (L. Santana Cabrera).
} 
and outside of service, to secure greater understanding of the processes of the organization and control of risk, to delimit responsibilities clearly to all the personnel, to make better use of the time and resources and, finally, to improve the motivation of the personnel.

(c) 2009 Elsevier España, S.L. and SEMICYUC. All rights reserved.

\section{Introducción}

La calidad asistencial tiene la finalidad de conseguir los siguientes objetivos: mejorar las prácticas sanitarias, lograr que el usuario esté satisfecho con la atención recibida, asegurar que estos cuidados sean accesibles y continuos, emplear intervenciones apropiadas a las necesidades de los pacientes $y$, por último, intentar obtener el máximo beneficio para su salud empleando el mínimo de recursos disponibles $^{1}$. No hay que confundir, en absoluto, el concepto de gestión de la calidad con el de gestión del gasto sanitario $^{2-4}$.

El mantenimiento de la calidad, tanto referida a la seguridad como a la eficacia, puede llevarse a cabo mediante un sistema de calidad revisado periódicamente por una certificación externa, según una norma, como la ISO (Internacional Organization for Standardization) ${ }^{5}$ 9001, cuya primera versión corresponde al año 1987; actualmente se encuentra en vigor la ISO 9001:2008, que hace especial énfasis en el control del cumplimiento de los requisitos legales aplicables al servicio que ofrece la organización certificada. Existen, aproximadamente, un millón de empresas certificadas según ISO 9001 en todo el mundo, y España, con unas 65.000 empresas certificadas, se encuentra en el cuarto lugar, por delante de países como Alemania, Estados Unidos o Gran Bretaña.

Los sistemas de gestión de la calidad son una herramienta muy potente y permiten priorizar acciones para que, con los mismos recursos, se puedan mantener la seguridad y la eficacia de las tecnologías sanitarias. Sobre la base de estas premisas, la Unidad de Cuidados Intensivos ( $\mathrm{UCl}$ ) de nuestro hospital llevó a cabo un proyecto de gestión de la calidad, y consiguió finalmente la certificación ISO 9001:2008 por el sistema de gestión y organización implantado en su servicio.

\section{Desarrollo del proceso de implementación de la norma ISO 9001:2008}

Tratamos de describir el trabajo realizado en nuestro servicio en relación con el proceso de implementación de un plan de gestión de la calidad, éste ha obtenido el reconocimiento como "Servicio Certificado que gestiona sus actividades según la norma UNE-EN ISO 9001:2008”.

Nuestra UCl se encuentra en un hospital terciario y forma parte de un complejo hospitalario, con una capacidad de 425 camas y 24 camas de cuidados intensivos polivalentes (medicoquirúrgicos y coronarios), está dividida en 3 módulos de 8 camas, dentro de unas instalaciones modernas, inauguradas en el año 2001, con una plantilla compuesta de un Jefe de Servicio, 16 médicos especialistas, 9 médicos residentes, un supervisor de enfermería y 74 enfermeros/as, con una ratio paciente/enfermero de $2 / 1$, y que oferta una amplia cartera de servicios (tabla 1).

La UCl tiene como finalidad prestar un servicio asistencial con calidad en el diagnóstico y el tratamiento que satisfaga al paciente crítico del sector de la población asignado y a sus familiares, con una cartera de servicios lo más integral y coordinada posible con otros servicios del complejo hospitalario, con el servicio de urgencias extrahospitalario (112) y con el resto de hospitales a los que sirve de referencia. La prestación de estos servicios se realiza con criterios de eficiencia, seguridad y bienestar, y se basa en un modelo de gestión de la calidad que garantiza los principios de universalidad, accesibilidad, equidad y solidaridad; para esto, se implementa un modelo que garantiza el cumplimiento de los requisitos de la norma UNE-EN ISO 9001, enfocado a la mejora continua, la legislación y la normativa del sector.

Las técnicas que se aplican, la disponibilidad de apoyo de otros especialistas, el uso del equipo apropiado, la implementación del seguimiento y la medición y de actividades de liberación y entrega se describen en el procedimiento Prestación de servicio de Medicina Intensiva.

Las técnicas se desarrollan según protocolos asistenciales o protocolos de tratamientos y de cuidados, según corresponda.

Es importante señalar que los documentos recogen el consenso entre facultativos, enfermería y las buenas prácticas de la evidencia científica, y que, al aprobarlos la Jefatura de la UCI, evidencian su aceptación y aplicación.

En ocasiones, el diagnóstico final se realiza a través de los resultados dados por otros servicios/unidades o la técnica se aplica fuera del recinto del servicio. La solicitud, el seguimiento y la aceptación de las prestaciones de otros servicios/unidades se recogen en el procedimiento Interrelación entre servicios/unidades.

Tabla 1 Cartera de servicios

1. Gestión asistencial a los trastornos cardiovasculares críticos

2. Gestión asistencial a los problemas respiratorios críticos

3. Gestión asistencial a los problemas metabólicos o hidroelectrolíticos

4. Gestión asistencial ante traumatismos graves

5. Gestión asistencial al postoperado de alto riesgo

6. Gestión asistencial al paciente con problemas neurológicos

7. Gestión asistencial a la paciente ginecológica crítica

8. Gestión asistencial al pretrasplante y al postrasplante de órganos 
Mapa de procesos genral

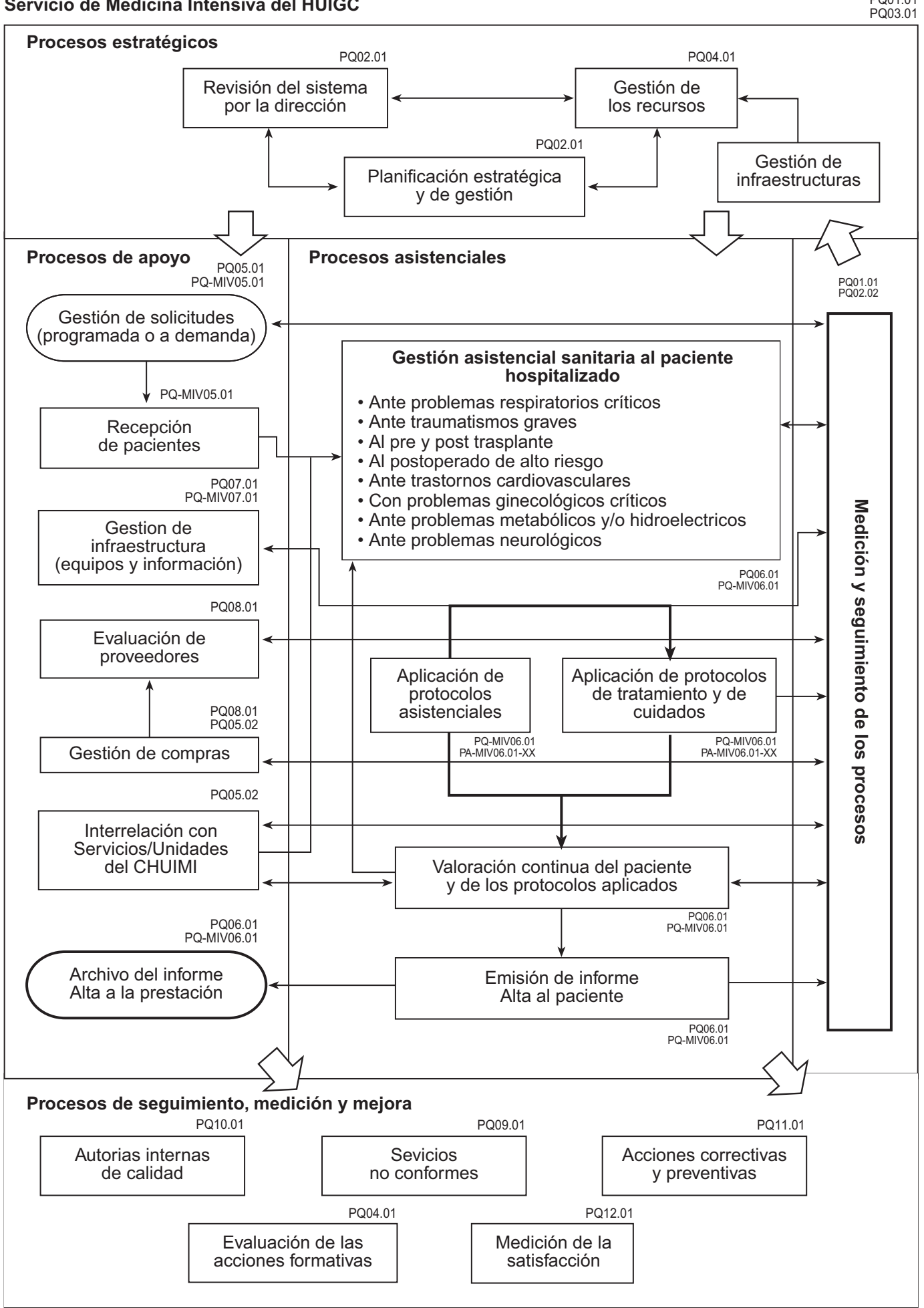

Figura 1 Mapa de procesos general.

Los recursos e infraestructuras se gestionan a través de los procedimientos Gestión de la infraestructura y almacén e Interrelación entre servicios/unidades.

El informe final se emite según se describe en el procedimiento Prestación de servicio de Medicina Intensiva, que se incluye en la historia clínica del paciente, y una copia del informe del alta médica se archiva en Administración.

Para visualizar la interacción entre los diferentes procesos definidos por la $\mathrm{UCI}$ se desarrolla un mapa de procesos, en el que se muestra cómo éstos interactúan con los procedimientos generales del complejo hospitalario (fig. 1). 
El sistema documental del servicio se fue desarrollando a medida que se fueron aprobando los procedimientos, los protocolos, etc. Se tuvo especial interés en describir las técnicas (protocolos asistenciales) que aplicamos y se consideraron en todos los casos los siguientes aspectos: denominación de la prestación, información previa requerida (incluye la existencia del consentimiento informado, sedación, analgesia aplicada o asepsia), técnica que se aplica, descripción de la técnica empleada, equipamiento e instrumental utilizado, limitaciones de la técnica empleada, resultados que se pueden obtener y, por último, los criterios de validación y rechazo.

Otros aspectos contemplados por el sistema de gestión, tales como el control de los documentos y registros, la revisión por parte de la Dirección, el análisis de datos, los indicadores, los recursos humanos, las auditorías internas, las no conformidades, las acciones correctivas, las acciones preventivas y la medición de la satisfacción se gestionan según se describe en el Manual de calidad. La aplicación de los aspectos anteriores se refleja en la definición de indicadores, tanto de gestión como de resultados para el servicio.

La relación de todos los procedimientos documentados del servicio, desde el punto de vista de gestión interno, queda reflejada en la tabla 2.

Las técnicas aplicadas y los criterios que usa la UCI según la enfermedad asistencial se documentan en Protocolos asistenciales (véase tabla 3).

Por último, tras todo este proceso de elaboración, se impartieron charlas sobre la aplicación de la ISO 9001, tanto al personal médico como de enfermería, y con los cursos recibidos se evaluó la formación sobre la base de criterios globales. Se creó la figura del responsable de calidad en el servicio y del responsable de la vigilancia de la infección. Se realiza un seguimiento a las no conformidades y se llevan a cabo acciones preventivas y correctivas. Asimismo, se realizan auditorías internas con personal asistencial y no asistencial de otros servicios, que lleva a cabo la unidad de calidad del complejo hospitalario.

Se analizan las reclamaciones a través de los canales establecidos y se realizan encuestas de satisfacción al paciente, a los familiares, al personal que trabaja en el servicio y al resto del personal del hospital con el que se mantiene una relación asistencial habitual.

Todo el funcionamiento del sistema de gestión en el servicio se describe en una memoria anual que se presenta a la Dirección de Gerencia para su valoración y respuesta.

Como se puede apreciar, de un enfoque meramente asistencial se han aplicado las herramientas de gestión que nos ofrece la norma internacional, y se ha velado en todo momento por la satisfacción de nuestros pacientes/usuarios.

\section{Discusión}

Durante los últimos años, los gobiernos de diferentes naciones han realizado un esfuerzo importante en aplicar principios de mejora de la calidad a la práctica de la medicina, en especial en el área de los cuidados intensivos. La calidad debe cumplir como objetivos no sólo la satisfacción de las expectativas de los pacientes y

Tabla 2 Lista de referencia principal del servicio

\begin{tabular}{ll}
\hline Códigos & Denominación \\
\hline MQ-MIV Anexo I & Declaración del Servicio de Medicina Intensiva del HUIGC \\
MQ-MIV Anexo II & Organigrama de la organización \\
MQ-MIV Anexo III & Mapa de procesos de la organización \\
PQ-MIV05.01 & Gestión de la cartera de servicio \\
PQ-MIV06.01 & Prestación de servicio \\
IT-MIV06.01-01 & Manual de uso del aplicativo informático \\
PQ-MIV06.01 Anexo I & Ingreso sin disponibilidad de cama en la UMI \\
PQ-MIV06.01 Anexo II & Grupos asistenciales \\
PQ-MIV06.01 Anexo III & Perfiles de solicitud a laboratorio \\
PQ-MIV06.01 Anexo IV & Orden de evacuación en caso de urgencia \\
PQ-MIV06.01 Anexo V & Normas de funcionamiento interno para el personal facultativo \\
PQ-MIV06.01 Anexo VI & Procesamiento de muestras biológicas \\
PQ-MIV06.01 Anexo VII & Sistema de clasificación de gravedad de enfermedad APACHE II \\
PQ-MIV06.01 Anexo VIII & Rotación antibiótica empírica \\
PQ-MIV06.01 Anexo IX & Criterios de diagnóstico de muerte encefálica \\
PQ-MIV06.01 Anexo X & Uso de dietas enterales \\
PQ-MIV06.01 Anexo XI & Motivo de ingresos y problemas \\
PQ-MIV06.01 Anexo XII & Composición de las nutriciones parenterales estándares \\
PQ-MIV06.01 Anexo XIII & Cuidados de enfermería ante un ingreso \\
PQ-MIV06.01 Anexo XIV & Criterios clínicos para la solicitud de radiografías de tórax portátiles \\
PQ-MIV06.01 Anexo XV & CID score \\
PQ-MIV06.01 Anexo XVI & Candida score \\
PQ-MIV07.01 & Gestión de las infraestructuras y almacén \\
\hline &
\end{tabular}

APACHE II: Acute Physiology and Chronic Health Evaluation; Candida score: sistema de puntuación predictor de candidiasis invasiva; CID: coagulación intravascular diseminada; HUIGM: Hospital Universitario Insular de Gran Canaria; UMI: unidad de Medicina Intensiva. 
Tabla 3 Protocolos asistenciales

\begin{tabular}{|c|c|}
\hline Códigos & Denominación \\
\hline PA-MIV06.01-01 & RCP básica y avanzada. Cuidados post-RCP \\
\hline PA-MIV06.01-02 & Monitorización cardíaca y hemodinámica básica \\
\hline PA-MIV06.01-03 & Monitorización cardíaca avanzada y asistencia circulatoria mecánica (medición del gasto cardíaco) \\
\hline PA-MIV06.01-04 & Capacidad de transporte secundario intrahospitalario asistido \\
\hline PA-MIV06.01-05 & Monitorización cardíaca avanzada y asistencia circulatoria mecánica (Swang-Ganz) \\
\hline PA-MIV06.01-06 & Monitorización cardíaca avanzada y asistencia circulatoria mecánica (Bciao) \\
\hline PA-MIV06.01-07 & Monitorización computarizada de trastornos del ritmo cardíaco \\
\hline PA-MIV06.01-08 & Control y tratamiento de la arritmia cardíaca grave con inestabilidad hemodinámica \\
\hline PA-MIV06.01-09 & Cardioversión sincronizada \\
\hline PA-MIV06.01-10 & Implantación de marcapasos temporal \\
\hline PA-MIV06.01-11 & Monitorización y asistencia al paciente afectado de síndrome coronario agudo \\
\hline PA-MIV06.01-12 & Tratamiento fibrinolítico en la cardiopatía isquémica \\
\hline PA-MIV06.01-13 & Monitorización de la función del intercambio gaseoso \\
\hline PA-MIV06.01-14 & Monitorización de la mecánica respiratoria \\
\hline PA-MIV06.01-15 & Instauración y mantenimiento de la vía respiratoria artificial (cualquier tipo) \\
\hline PA-MIV06.01-16 & Soporte ventilatorio artificial con o sin apoyo de sedorrelajación (VMI) \\
\hline PA-MIV06.01-17 & Soporte ventilatorio no invasivo \\
\hline PA-MIV06.01-18 & Fibrobroncoscopia terapéutica \\
\hline PA-MIV06.01-19 & Capacidad de implantación y mantenimiento de drenajes pleurales \\
\hline PA-MIV06.01-20 & Capacidad de implantacion y mantenimiento de drenajes pericárdicos \\
\hline PA-MIV06.01-21 & Equilibrio acidobásico e hidroelectrolítico \\
\hline PA-MIV06.01-22 & Nutrición enteral \\
\hline PA-MIV06.01-23 & Control y mantenimiento del soporte nutricional parenteral \\
\hline PA-MIV06.01-24 & Asistencia avanzada al fracaso multiorgánico \\
\hline PA-MIV06.01-25 & Asistencia al neurotrauma inicial y durante períodos prolongados y con monitorización avanzada \\
\hline PA-MIV06.01-26 & Monitorización neurológica: DTC \\
\hline PA-MIV06.01-27 & Monitorización Neurológica: PIC intraparerenquimatosa \\
\hline PA-MIV06.01-28 & Monitorización neurológica: PIC intraventricular \\
\hline PA-MIV06.01-29 & Soporte hemodinámico farmacológico \\
\hline PA-MIV06.01-30 & Atención avanzada al politraumatizado \\
\hline PA-MIV06.01-31 & Atención al postoperado de alto riesgo \\
\hline PA-MIV06.01-32 & Atención al paciente quemado \\
\hline PA-MIV06.01-33 & Mantenimiento del donante \\
\hline PA-MIV06.01-34 & Atención posquirúrgica del trasplantado \\
\hline PA-MIV06.01-35 & Depuración extrarrenal continua e intermitente \\
\hline PA-MIV06.01-36 & Cateterización venosa y arterial \\
\hline Anexo I del PA-MIV06.01-36 & Lista de verificación en la inserción de vías centrales \\
\hline
\end{tabular}

DTC: Doppler transcraneal; PIC: presión intracraneal; RCP: reanimación cardiopulmonar; VMl: ventilación mecánica invasiva.

familiares (calidad percibida), sino también la efectividad y eficiencia en la actuación (calidad intrínseca, cientificotécnica).

La norma UNE-EN ISO 9001 especifica los requisitos de un sistema de gestión de la calidad. Se trata de una norma genérica, aplicable a cualquier tipo de organización, que en los últimos años está teniendo una amplia difusión en el ámbito sanitario. Está basada en 8 principios de gestión de la calidad, entre los que destacan la orientación al cliente, el enfoque a procesos y la mejora continua. Es una herramienta de gestión que se centra en lograr la satisfacción de los clientes con los servicios que reciben, a través de un control eficaz de los procesos y de la búsqueda sistemática de oportunidades de mejora.
La acreditación administrativa de centros o servicios sanitarios es un proceso de evaluación por parte de la autoridad sanitaria que analiza la capacidad de un centro sanitario para su apertura. Esta evaluación, que incluye la revisión de su sistema de trabajo, se lleva a cabo en el momento de inicio de actividad del centro, pero no asegura el continuo cumplimiento de los requisitos por parte de éste durante el tiempo. Para lograrlo, se debe recurrir a un sistema de gestión que lo asegure y que, naturalmente, incluya auditorías periódicas.

Como cualquier herramienta, la certificación tiene unos costes de puesta en marcha que deben verse como una inversión y no como un gasto. Actualmente, en los centros sanitarios se apuesta por los sistemas de gestión de la 
calidad, pues la labor de la unidad de gestión de la calidad hospitalaria ha sido muy efectiva en cuanto al control y, sobre todo, al rigor; da credibilidad al sistema y aporta ventajas en la gestión.

Es importante disponer de un canal de comunicación entre los proveedores de la salud, ya que los fallos en esta comunicación pueden llevar a provocar daños en el paciente. Una herramienta disponible son los daily goals checklist, o lista de comprobación diaria de objetivos, que debe cumplimentar cada facultativo/enfermera y que producen una mejoría en la comunicación entre el equipo de profesionales que atiende al paciente y los miembros de la familia en relación con el plan de cuidados ${ }^{6}$.

En la actualidad, no hay un sistema o modelo de gestión de la calidad único de aplicación en el sector sanitario, y se pueden aplicar la EFQM (European Foundation for Quality Model) ${ }^{7}$, la Norma ISO $^{5}$ o la JCAHO (Joint Comisión on Accreditation of Healthcare Organizations) ${ }^{8}$, entre otros. Los ejemplos anteriores tienen en común que se crearon para la mejora de los resultados de las organizaciones, impulsan la mejora continuada a través de la aplicación del ciclo PDCA (plan, do, check, action), están relacionados con la calidad, requieren el compromiso de la dirección, están orientados al cliente y sometidos a evaluaciones externas ${ }^{9}$.

En esta línea, la Sociedad Española de Medicina Intensiva Crítica y Unidades Coronarias (SEMICYUC), en colaboración con la Fundación Avedis Donabedian (FAD), publicó el libro titulado "Indicadores de calidad en el enfermo crítico", donde se elaboraron 120 indicadores; de éstos, 20 se consideraron relevantes ${ }^{10}$.

La evaluación de la calidad asistencial se realiza mediante indicadores. Deben tenerse en cuenta indicadores de gestión, de resultados y de satisfacción.

Los indicadores de estructura miden cómo está organizado y equipado el sistema de salud, y observan si los recursos están disponibles y en condiciones para facilitar la atención al usuario; evalúan los recursos (humanos, materiales, tecnológicos y financieros), la estructura organizativa y cómo las $\mathrm{UCl}$ se integran en el hospital.

Los indicadores de proceso evalúan la forma en que se desarrolla la práctica asistencial, realizada con los recursos disponibles, protocolos y evidencia científica. Se asume que los datos relacionados con la evaluación del proceso son más sensibles como indicadores de calidad que los relacionados con los resultados, ya que un resultado adverso no siempre se produce cuando existen errores en el proceso ${ }^{11}$. De todas formas, la detección de efectos adversos, aunque sean pequeños, ofrece importantes oportunidades de mejora en la asistencia, y las $\mathrm{UCl}$ pueden ser un auténtico observatorio, no sólo de los errores propios, sino también de los causados en otros servicios del hospital ${ }^{12}$.

Los indicadores de resultado miden las consecuencias del proceso asistencial en términos de complicaciones, mortalidad, ocasiones perdidas, fallos de circuitos, calidad de vida, etc.

La certificación, según la UNE-EN ISO 9001 obtenida, supone la garantía de que los procedimientos llevados a cabo en el servicio en lo referente a la gestión de procesos y la formación de personal siguen las pautas marcadas en el sistema y que no variarán, independientemente de quién los ejecute y del tiempo que lleve trabajando en el servicio. Con la certificación ISO 9001 se acredita un sistema de calidad que tiene como fin la satisfacción del cliente, que en este caso son los enfermos, sus familiares y el personal de otros servicios que piden nuestra ayuda en algún momento. Esto no significa que ahora trabajemos con más calidad, sino que ya existe la seguridad de que el trabajo está bien hecho y de que no hay variabilidad en los profesionales aunque haya sustituciones, además de que mejoraremos año tras año porque estamos obligados a ello.

En conclusión, los beneficios de la aplicación de un sistema de gestión de calidad incluyen, entre otros, detectar las necesidades que puede cubrir el servicio con el fin de lograr la satisfacción del paciente, sus familiares o el personal sanitario del resto de los servicios del hospital, mejorar las comunicaciones dentro y fuera del servicio, conseguir una mayor comprensión de los procesos de la organización y el control de riesgo, delimitar responsabilidades de forma clara a todo el personal, llevar a acabo una mejor utilización del tiempo y los recursos y, por último, mejorar, si cabe, la motivación del personal. Por tanto, el esfuerzo de implementar sistemas de análisis de la calidad redundará en beneficio de todos los actores del sistema sanitario, ciudadanos, profesionales, gestores y financiadores ${ }^{13}$.

\section{Agradecimiento}

Los autores desean agradecer la colaboración para conseguir la Certificación UNE-EN ISO 9001:2008 a todo el personal que trabaja en la UCl y que participó en la elaboración de la documentación, así como a la Unidad de Gestión de la Calidad de Complejo Hospitalario.

\section{Bibliografía}

1. Soto Álvarez J. Implicación de la investigación de resultados en salud en la mejora continua de la calidad asistencial del Sistema Nacional de Salud. An Med Interna (Madrid). 2007;24:517-19.

2. Barrientos R, Morales C, Robas A. Costes de un Servicio de Cuidados Intensivos. Med Intensiva. 1993;17:40-6.

3. Ortún Rubio V, Meneu de Guillerna R. The impact of economics on health policy and management in Spain. Rev Esp Salud Pública. 2006;80:491-504.

4. Curtis JR, Cook DJ, Wall RJ, Angus DC, Bion J, Kackmarek R, et al. Intensive care unit quality improvement: A "how-to" guide for the interdisciplinary team. Crit Care Med. 2006;34:211-18.

5. Página oficial de la International Organization for Standardization. [citado 3 Jun 2009]. Disponible en: URL: http://www.iso.org/iso/home.htm.

6. Pronovost PJ, Berenholtz S, Dorman T, Lipsett P, Simmonds T, Haraden C. Improving communication in the ICU using daily goals. J Crit Care. 2003;18:71-5.

7. Página oficial de la European Foundation for Quality Management. [citado 3 Jun 2009]. Disponible en: URL: http://ww1. efqm.org/en/.

8. Página oficial de la Joint Comisión on Accreditation of Healthcare Organizations (JCAHO). [citado 3 Jun 2009]. Disponible en: URL: http://www.jointcommission.org/.

9. Davins Miralles J. Comparativa de 3 modelos de gestión de calidad: EFQM, ISO, JCAHO. FMC. 2007;14:328-32.

10. Sociedad Española de Medicina Intensiva Crítica y Unidades Coronarias. Indicadores de calidad en el enfermo crítico. $1 .^{\mathrm{a}}$ ed. Madrid; 2005 [citado 10 May 2009]. Disponible en: http: / /www. calidad.semicyuc.org. 
11. Martín MC, Cabré L, Ruiz J, Blanch L, Blanco J, Castillo F, et al. Grupos de trabajo de la Sociedad Española de Medicina Intensiva Crítica y Unidades Coronarias (SEMICYUC), Sociedad Española de Enfermería Intensiva y Unidades Coronarias (SEEIUC) y Fundación AVEDIS Donabedian (FAD). Indicadores de calidad en el paciente crítico. Med Intensiva. 2008;32:23-32.
12. Hernández García I, González Celador R, Sáenz González MC. Características de los efectos adversos detectados en una unidad de cuidados intensivos de adultos. Rev Calidad Asistencial. 2008;23:150-7.

13. Carrasco G, Pallarés A, Cabré L. Costes de la calidad en Medicina Intensiva. Guía para gestores clínicos. Med Intensiva. 2006;30:167-79. 